\title{
COMPOSITION OPERATORS ON HARDY-ORLICZ SPACES ON THE BALL
}

\author{
STÉPHANE CHARPENTIER
}

\begin{abstract}
We give embedding theorems for Hardy-Orlicz spaces on the ball and then apply our results to the study of the boundedness and the compactness of composition operators in this context. As one of the motivations of this work, we show that there exist some Hardy-Orlicz spaces, different from $H^{\infty}$, on which every composition operator is bounded.
\end{abstract}

\section{INTRODUCTION AND PRELIMINARIES}

1.1. Introduction. The continuity and the compactness of composition operators $C_{\phi}$, defined by $C_{\phi}(f)=f \circ \phi$, on usual analytic functions spaces have been studied in different ways. On Hardy spaces $H^{p}\left(\mathbb{B}_{N}\right)$ and Bergman spaces $A^{p}\left(\mathbb{B}_{N}\right), 1 \leq p<\infty$, they have been characterized for instance in terms of Carleson measures [4]. In one variable, the Littlewood subordination principle permits to show that the Carleson necessary and sufficient condition which deals with the boundedness of $C_{\phi}$ on these spaces is always satisfied, i.e. that every composition operator is bounded in this context ([16]). Anyway, it appears that both boundedness and compactness of $C_{\phi}$ on $H^{p}\left(\mathbb{B}_{N}\right)$ (resp. $A^{p}\left(\mathbb{B}_{N}\right)$ ) is independent of $p$. On the other side, it is not difficult to check that $C_{\phi}$ is compact on $H^{\infty}$ if and only if $\|\phi\|_{\infty}<1$, so that there is a "break of condition" between $H^{\infty}$ and $H^{p}(\mathbb{D})\left(\operatorname{resp} . A^{p}(\mathbb{D})\right.$ ), regarding to the compactness of $C_{\phi}$.

This observation motivated P. Lefèvre, D. Li, H. Queffélec and L. Rodríguez-Piazza to study composition operators on Hardy-Orlicz spaces $H^{\psi}(\mathbb{D})$ (resp. Bergman-Orlicz spaces $A^{\psi}(\mathbb{D})$ ) of the disk which are an intermediate scale of spaces between $H^{\infty}$ and $H^{p}\left(\mathbb{B}_{N}\right)$ (resp. $A^{p}(\mathbb{D})$ ) and also generalize these classical Hardy (resp. Bergman) spaces. Since 2006, they produced some papers on this subject, e.g. [9, 10, 7, 8], in which they gave characterizations of boundedness and compactness of $C_{\phi}$ on these spaces, for instance. Moreover, they have been interesting in the question of the existence of some Hardy-Orlicz spaces on which the compactness of $C_{\phi}$ is equivalent to that on $H^{\infty}$. Yet, they answer this question to the negative, by proving in [10, Theorem 4.1] that, for every Hardy-Orlicz space $H^{\psi}(\mathbb{D})$, we can construct a surjective map $\phi: \mathbb{D} \rightarrow \mathbb{D}$ which induces a composition operator $C_{\phi}$ which is compact on $H^{\psi}(\mathbb{D})$. In fact, this result extends that obtained by B. MacCluer and J. Shapiro in the context of classical Hardy spaces ([12, Example 3.12]). The same problem in Bergman-Orlicz framework has not been completely solved yet.

In the several-variables setting, this motivation appears to be even more important, since it concerns continuity and not only compactness. Indeed, it is well-known that there exist symbols $\phi$ such that $C_{\phi}$ is not bounded on the classical Hardy spaces $H^{p}\left(\mathbb{B}_{N}\right.$ ) (resp. Bergman spaces $A^{p}\left(\mathbb{B}_{N}\right)$ ), although every $C_{\phi}$ is bounded on $H^{\infty}$. Precisely, one can ask the question: is there some growth or regular condition on $\psi$ in order that every composition operator is bounded on such $H^{\psi}\left(\mathbb{B}_{N}\right)$ (resp. $A^{\psi}\left(\mathbb{B}_{N}\right)$ ). This question has been treated in [3], in which paper characterizations of boundedness and compactness of $C_{\phi}$ on weighted

2000 Mathematics Subject Classification. Primary: 47B33 - Secondary: 32C22; 46E15.

Key words and phrases. Carleson measure - Composition operator - Hardy-Orlicz space. 
Bergman-Orlicz spaces are given in satisfying generality. It reveals that the answer to the question above is yes in this framework. The present paper intends to give similar results in the context of Hardy-Orlicz spaces.

It is organized as follows: after recalling the definition of Hardy-Orlicz spaces and introducing the materials involved, we conclude the first section by giving some topological and duality results on Hardy-Orlicz spaces. Section 2 is devoted to general adapted Carleson embedding theorems, which are the main tools to get, in Section 3, characterizations of boundedness and compactness of composition operators on these spaces. We will have to face some difficulties in applying our Carleson embedding theorems, due to the fact that Hardy-Orlicz spaces are not separable in general. We finish the section with some consequences of these characterizations, especially the exhibition of a class of Orlicz functions $\psi$ defining Hardy-Orlicz spaces $H^{\psi}\left(\mathbb{B}_{N}\right)$, on which every composition operator is bounded. It turns out that this condition is the same than that given for Bergman-Orlicz spaces in [3].

\subsection{Orlicz spaces - Notations.}

1.2.1. Definitions. A strictly convex function $\psi: \mathbb{R}_{+} \rightarrow \mathbb{R}_{+}$is called an Orlicz function if $\psi(0)=0, \psi$ is continuous at 0 and $\frac{\psi(x)}{x} \underset{x \rightarrow+\infty}{\longrightarrow}+\infty$. If $(\Omega, \mathbb{P})$ is a probability space, the Orlicz space $L^{\psi}(\Omega)$ associated to the Orlicz function $\psi$ on $(\Omega, \mathbb{P})$ is the set of all (equivalence classes of) measurable functions $f$ on $\Omega$ such that there exists some $C>0$, such that $\int_{\Omega} \psi\left(\frac{|f|}{C}\right) d \mathbb{P}$ is finite. $L^{\psi}(\Omega)$ is a vector space, which can be normed with the so-called Luxemburg norm defined by

$$
\|f\|_{\psi}=\inf \left\{C>0, \int_{\Omega} \psi\left(\frac{|f|}{C}\right) d \mathbb{P} \leq 1\right\}
$$

in this case, it is well-known that $\left(L^{\psi}(\Omega),\|\cdot\|_{\psi}\right)$ is a Banach space.

It comes from the definition that, for every Orlicz function $\psi$, we have the inclusions $L^{\infty} \subset L^{\psi}(\Omega) \subset L^{p}(\Omega)$. Moreover, if $\psi(x)=x^{p}$, for some $1 \leq p<\infty$ and for every $x \geq 0$, then $L^{\psi}(\Omega)$ coincides with the usual Lebesgue space $L^{p}(\Omega)$.

We also introduce the Morse-Transue space $M^{\psi}(\Omega)$ as the subspace of $L^{\psi}(\Omega)$ generated by $L^{\infty}(\Omega)$, and for every Orlicz function $\psi$, we can consider its complementary function $\Phi$ defined by $\Phi(y)=\sup _{x \in \mathbb{R}_{+}}\{x y-\psi(x)\}$, which can be shown to be an Orlicz function too. These two notions permit to identify (isomorphically) the dual space $\left(M^{\psi}(\Omega)\right)^{*}$ of $M^{\psi}(\Omega)$ and $L^{\Phi}$, whenever both of these two spaces are normed with the Luxemburg norm, with the natural integral duality crochet (see e.g. [14, IV, 4.1, Theorem 7]).

1.2.2. Four classes of Orlicz functions. In order to distinguish the Orlicz spaces and to get a meaningful scale of intermediate such spaces between $L^{\infty}$ and $L^{p}(\Omega)$, we use to classify the Orlicz functions, with respect to their growth or their regularity. In this paper, as in [3], we introduce essentially four classes of Orlicz spaces.

- The first class is that of Orlicz functions which satisfy the so-called $\Delta_{2}$-Condition which is a condition of moderate growth: an Orlicz function $\psi$ satisfies the $\Delta_{2}$-Condition if there exist $x_{0}>0$ and a constant $K>1$, such that $\psi(2 x) \leq K \psi(x)$ for any $x \geq x_{0}$.

For example, $x \longmapsto a x^{p}(1+b \log (x)), p>1, a>0$ and $b \geq 0$, satisfies the $\Delta_{2^{-}}$ Condition. Corollary 5, Chapter II of [14] reads:

Proposition 1.1. Let $\psi$ be an Orlicz function satisfying the $\Delta_{2}$-Condition. Then there are some $p>1$ and $C>0$ such that $\psi(x) \leq C x^{p}$, for $x$ large enough. Therefore, $L^{p} \subset L^{\psi} \subset L^{1}$, for some $p>1$. 
- The $\nabla_{2}$-class contains the Orlicz functions whose complementary ones $\Phi$ satisfy the $\Delta_{2^{-}}$ condition. It is a condition of regularity and it is equivalent to the existence of some $\beta>1$ and some $x_{0}>0$, such that $\psi(\beta x) \geq 2 \beta \psi(x)$, for $x \geq x_{0}$.

We need to mention that, if both $L^{\psi}(\Omega)$ and $L^{\Phi}(\Omega)$ are normed by the Luxemburg norm, then $L^{\psi}(\Omega)$ is isomorphic to the dual of $L^{\Phi}(\Omega)$, as soon as $\psi$ satisfies the $\nabla_{2^{-}}$ Condition. Moreover, we have the following interpolation theorem, which is not general, but which will be sufficient for our purpose. It is nothing but [9, Proposition 3.5]:

Proposition 1.2. Let $\psi$ be an Orlicz function which satisfies the $\nabla_{2}$-Condition. Then every linear, or sub-linear, operator which is of weak-type $(1,1)$ and strong type $(\infty, \infty)$ is bounded from $L^{\psi}$ into $L^{\psi}$.

- The two following conditions are also regular conditions which are satisfied by most of the Orlicz functions that we are interesting in: $\psi$ satisfies the $\nabla_{0^{-}}$Condition if there exist some $x_{0}>0$ and some constant $C \geq 1$, such that for every $x_{0} \leq x \leq y$ we have

$$
\frac{\psi(2 x)}{\psi(x)} \leq \frac{\psi(2 C y)}{\psi(y)}
$$

Proposition 4.6 of [9] ensures that this latter condition is still equivalent to the fact that, for every (or equivalently one) $\beta>1$, there exists a constant $C_{\beta} \geq 1$ such that

$$
\frac{\psi(\beta x)}{\psi(x)} \leq \frac{\psi\left(\beta C_{\beta} y\right)}{\psi(y)}
$$

for every $x_{0} \leq x \leq y$.

We shall consider the following subclass: $\psi$ satisfies the uniform $\nabla_{0}$-Condition if it satisfies the $\nabla_{0}$-Condition for a constant $C_{\beta} \geq 1$ independent of $\beta>1$.

- Finally, one defines a class of Orlicz functions which grow fast: $\psi$ satisfies the $\Delta^{2}$ Condition if there exist $x_{0}>0$ and a constant $C>0$, such that $\psi(x)^{2} \leq \psi(C x)$ for every $x \geq x_{0}$.

The convexity and the non-decrease of Orlicz functions give the following proposition ([14, Chapter II, Paragraph 2.5, pages 40 and further] or [6, Chapter I, Section 6, Paragraph 5]):

Proposition 1.3. Let $\psi$ be an Orlicz function. The assertions:

(1) $\psi$ satisfies the $\Delta^{2}$-Condition;

(2) There exist $b>1, C>0$ and $x_{0}>0$ such that $\psi(x)^{b} \leq \psi(C x)$, for every $x \geq x_{0}$;

(3) For every $b>1$, there exist $C_{b}>0$ and $x_{0, b}>0$ such that $\psi(x)^{b} \leq \psi\left(C_{b} x\right)$, for every $x \geq x_{0, b}$.

are equivalent.

[14, Chapter II, Paragraph 2, Proposition 6]) says that an Orlicz function which satisfies the $\Delta^{2}$-Condition need to have at least an exponential growth:

Proposition 1.4. Let $\psi$ be an Orlicz function which satisfies the $\Delta^{2}$-Condition. There exist $a>0$ and $x_{0}>0$ such that

$$
\psi(x) \geq e^{a x}
$$

for every $x \geq x_{0}$.

If $\psi$ satisfies $\Delta^{2}$-Condition, we shall say that $L^{\psi}(\Omega)$ is a "small" Orlicz space, i.e. "far" from any $L^{p}(\Omega)$ and "close" to $L^{\infty}$.

To finish, Proposition 4.7 (2) of [9] establishes relations between these conditions: 
Proposition 1.5. Let $\psi$ be an Orlicz function.

(1) If $\psi$ satisfies the uniform $\nabla_{0}$-Condition, then it satisfies the $\nabla_{2}$-Condition;

(2) If $\psi$ satisfies the $\Delta^{2}$-Condition, then it satisfies the uniform $\nabla_{0}$-Condition.

For any $1<p<\infty$, every function $x \longmapsto x^{p}$ is an Orlicz function which satisfies the uniform $\nabla_{0}$-Condition, (so $\nabla_{2}$ and $\nabla_{0}$-conditions too) and the $\Delta_{2}$-Condition. At the opposite side, for any $a>0$ and $b \geq 1, x \longmapsto e^{a x^{b}}-1$ belongs to the $\Delta^{2}$-Class (and then to the uniform $\nabla_{0}$-Class), yet not to the $\Delta_{2}$-one. In addition, the Orlicz functions which can be written $x \rightarrow \exp \left(a(\ln (x+1))^{b}\right)-1$ for $a>0$ and $b \geq 1$, satisfy the $\nabla_{2}$ and $\nabla_{0}$-Conditions, but do not belong to the $\Delta^{2}$-Class.

For a complete study of Orlicz spaces, we refer to [6] and [14]. We can also find precise and useful information in [9], such as other classes of Orlicz functions and their link together with.

1.3. Hardy-Orlicz spaces on $\mathbb{B}_{N}$. The definition of Hardy-Orlicz spaces on the ball is quite similar to that of classical Hardy spaces. With the notations above, $(\Omega, \mathbb{P})$ stands for $\left(\mathbb{S}_{N}, d \sigma_{N}\right)$, where $\mathbb{S}_{N}=\partial \mathbb{B}_{N}$ is the unit sphere of $\mathbb{C}^{N}$ and $d \sigma_{N}$ is the normalized Lebesgue measure on $\mathbb{S}_{N}$. Given an Orlicz function $\psi$, the Hardy-Orlicz space $H^{\psi}\left(\mathbb{B}_{N}\right)$ on $\mathbb{B}_{N}$ is the vector space of analytic functions $f: \mathbb{B}_{N} \rightarrow \mathbb{C}$ such that $\sup _{0<r<1}\left\|f_{r}\right\|_{\psi}<\infty$, where $f_{r} \in L^{\psi}\left(\mathbb{S}_{N}\right)$ is defined by $f_{r}(z)=f(r z)$.

Since $L^{\psi}\left(\mathbb{B}_{N}\right) \subset L^{1}\left(\mathbb{B}_{N}\right), H^{\psi}\left(\mathbb{B}_{N}\right)$ is contained in $H^{1}\left(\mathbb{B}_{N}\right)$. In particular, any $f \in$ $H^{\psi}\left(\mathbb{B}_{N}\right)$ admits a radial limit $f^{*}$ almost everywhere on $\mathbb{S}_{N}$, and we have the following theorem, which can be generalized from the well-known case $\psi(x)=x^{p}$ :

Theorem 1.6. Let $f \in H^{\psi}\left(\mathbb{B}_{N}\right)$ and let $f^{*}$ be its almost everywhere boundary limit. Then $f^{*} \in L^{\psi}\left(\mathbb{S}_{N}\right)$ and

$$
\left\|f^{*}\right\|_{\psi}=\sup _{0<r<1}\left\|f_{r}\right\|_{\psi} .
$$

If we denote by $\|f\|_{H^{\psi}}:=\left\|f^{*}\right\|_{\psi}$, then $H^{\psi}\left(\mathbb{B}_{N}\right)$ endowed with the norm $\|\cdot\|_{H^{\psi}}$ is a Banach space.

The proof of this result is quite identical to that in the one variable setting ([9, Proposition 3.1]). When there is no possible confusion, we will indifferently denote by $\|\cdot\|_{\psi}$ the norm $\|.\|_{H^{\psi}}$ on $H^{\psi}\left(\mathbb{B}_{N}\right)$ and we identify $H^{\psi}\left(\mathbb{B}_{N}\right)$ to a subspace of $L^{\psi}\left(\mathbb{S}_{N}\right)$. Similarly, if $f \in$ $H^{\psi}\left(\mathbb{B}_{N}\right.$ ), we will sometimes write $f^{*}$ (or even just $f$ ) the function equal to $f$ in $\mathbb{B}_{N}$ and equal to the boundary radial limits of $f$ almost everywhere on $\mathbb{S}_{N}$.

In the sequel, we will denote by $P_{z}$ the invariant Poisson kernel at $z \in \mathbb{B}_{N}$,

$$
P_{z}(\zeta)=P(z, \zeta)=\left(\frac{1-|z|^{2}}{|1-\langle z, \zeta\rangle|^{2}}\right)^{N}, z \in \mathbb{B}_{N}, \zeta \in \mathbb{S}_{N}
$$

We will also use the notation $u_{\zeta, r}, \zeta \in \mathbb{S}_{N}$ and $0<r<1$, for the function

$$
u_{\zeta, r}(z)=\left(\frac{1-r}{1-r\langle z, \zeta\rangle}\right)^{2 N}, z \in \overline{\mathbb{B}_{N}} .
$$

We easily check that $u_{\zeta, r} \in H^{\infty}$ and that $\left\|u_{\zeta, r}\right\|_{\infty}=1$ and . If $z \in \mathbb{S}_{N}$, then

$$
u_{\zeta, r}(z)=\left(\frac{1-r}{1+r}\right)^{N} P(r z, \zeta),
$$


so that $\left\|u_{\zeta, r}\right\|_{L^{1}\left(\mathbb{S}_{N}\right)}=\left(\frac{1-r}{1+r}\right)^{N}$.

As usual we shall verify that the point evaluation linear functional maps $\delta_{z}$ at $z \in \mathbb{B}_{N}$, defined by $\delta_{z}(f)=f(z)$ for $f \in H^{\psi}\left(\mathbb{B}_{N}\right)$ are bounded.

Proposition 1.7. If $z \in \mathbb{B}_{N}$ then

$$
\frac{1}{4^{N}} \psi^{-1}\left(\left(\frac{1+|z|}{1-|z|}\right)^{N}\right) \leq\left\|\delta_{z}\right\|_{\left(H^{\psi}\left(\mathbb{B}_{N}\right)\right)^{*}} \leq \psi^{-1}\left(\left(\frac{1+|z|}{1-|z|}\right)^{N}\right) .
$$

Proof. Since $H^{\psi}\left(\mathbb{B}_{N}\right) \subset H^{1}\left(\mathbb{B}_{N}\right)$, for every $f \in H^{\psi}\left(\mathbb{B}_{N}\right)$, we have

$$
f(z)=\int_{\mathbb{S}_{N}} P_{z}(\zeta) f(\zeta) d \sigma(\zeta)
$$

By Jensen's Inequality and because $\left\|P_{z}\right\|_{\infty} \leq\left(\frac{1+|z|}{1-|z|}\right)^{N}$, we get

$$
\psi\left(\left|\frac{f(z)}{\|f\|_{\psi}}\right|\right) \leq \int_{\mathbb{S}_{N}} P_{z}(\zeta) \psi\left(\frac{|f(\zeta)|}{\|f\|_{\psi}}\right) d \sigma(\zeta) \leq\left(\frac{1+|z|}{1-|z|}\right)^{N},
$$

hence

$$
|f(z)| \leq\|f\|_{\psi} \psi^{-1}\left(\left(\frac{1+|z|}{1-|z|}\right)^{N}\right) .
$$

For the lower bound, it is sufficient to compute $\delta_{z}\left(u_{\zeta, r}\right)$ and $\left\|u_{\zeta, r}\right\|_{\psi}$, thanks to [9, Lemme 3.9], with $r=|z|$ and $\zeta \in \mathbb{S}_{N}$ such that $\langle z, \zeta\rangle=r$. The details are left to the reader.

Contrary to what happens for the Hardy spaces, the ball algebra $A\left(\mathbb{B}_{N}\right)$ is not always dense in $H^{\psi}\left(\mathbb{B}_{N}\right)$, as the following result indicates (from now on, $H M^{\psi}\left(\mathbb{B}_{N}\right)$ denotes $H^{\psi}\left(\mathbb{B}_{N}\right) \cap M^{\psi}\left(\mathbb{B}_{N}\right)$.)

Theorem 1.8 (Chapter IX, Theorem 4 of [14] for $\Omega=\mathbb{B}_{N}$ ). Let $\psi$ be an Orlicz function. $A\left(\mathbb{B}_{N}\right)$ is dense in $H^{\psi}\left(\mathbb{B}_{N}\right)$ if and only if $H^{\psi}\left(\mathbb{B}_{N}\right)$ is separable, which in turn is equivalent to the fact that $\psi$ satisfies the $\Delta_{2}$-Condition. However, $H M^{\psi}\left(\mathbb{B}_{N}\right)$ is always separable.

The non-separability of $H^{\psi}\left(\mathbb{B}_{N}\right)$ will cause some problems when we will try to deduce results on composition operators from embedding theorems. Yet, we have the following result:

Theorem 1.9. Let $\psi$ be an Orlicz function.

(1) $H M^{\psi}\left(\mathbb{B}_{N}\right)$ is the closure of $H^{\infty}$ in $L^{\psi}\left(\mathbb{S}_{N}\right)$; in particular the set of all polynomials is dense in $H M^{\psi}\left(\mathbb{B}_{N}\right)$ for the topology defined by $\|\cdot\|_{\psi}$. More precisely, for every $f \in H M^{\psi}\left(\mathbb{B}_{N}\right),\left\|f_{r}-f\right\|_{\psi} \underset{r \rightarrow 1}{\longrightarrow} 0$.

(2) On the unit ball of $H^{\psi}\left(\mathbb{B}_{N}\right)$ (or equivalently on every ball), the induced weak-star topology coincides with that of uniform convergence on compacta of $\mathbb{B}_{N}$.

(3) $H^{\psi}\left(\mathbb{B}_{N}\right)$ is weak-star closed in $L^{\psi}\left(\mathbb{S}_{N}\right)$.

(4) If $\psi$ satisfies the $\nabla_{2}$-Condition, then $H^{\psi}\left(\mathbb{B}_{N}\right)$ can be isometrically identified to $\left(H M^{\psi}\left(\mathbb{B}_{N}\right)\right)^{* *}$. In particular, if $\psi$ satisfies $\nabla_{2}$ and $\Delta_{2}$-Conditions, then $H^{\psi}\left(\mathbb{B}_{N}\right)$ is reflexive.

Proof. 1) It suffices to show that $\left\|f_{r}-f\right\|_{\psi} \underset{r \rightarrow 1}{\longrightarrow} 0$ for every $f \in H M^{\psi}\left(\mathbb{B}_{N}\right)$; the proof of this fact is identical to that of [9, Proposition 3.4]. 
2) This is a classical use of Poisson kernel and Proposition 1.7.

3) It is sufficient to show that the balls of $H^{\psi}\left(\mathbb{B}_{N}\right)$ are weak-star closed, i.e. compact. Using point 2) and the fact that the topology of uniform convergence on compacta is metrizable, we are led to show that, if $\left(f_{n}\right)_{n}$ is a sequence in the unit ball of $H^{\psi}\left(\mathbb{B}_{N}\right)$, then we can extract from it a subsequence which converges uniformly on every compacta to some $f$ in the unit ball of $H^{\psi}\left(\mathbb{B}_{N}\right)$. Now this follows from Proposition 1.7, which ensures that $\left(f_{n}\right)_{n}$ is a normal family, and from Fatou's lemma.

4) Since $\left(M^{\psi}\left(\mathbb{S}_{N}\right)\right)^{*}=L^{\phi}\left(\mathbb{S}_{N}\right)$ and since $\left(L^{\phi}\left(\mathbb{S}_{N}\right)\right)^{*}=L^{\psi}\left(\mathbb{S}_{N}\right)$, because $\psi$ satisfies the $\nabla_{2}$-Condition $\left(\left[14, \mathrm{IV}, 4.1\right.\right.$, Corollary 9 , p. 111]), then $\left(M^{\psi}\left(\mathbb{S}_{N}\right)\right)^{* *}=L^{\psi}\left(\mathbb{S}_{N}\right)$. Now, using [5, V, 5.6, Corollary 6], we get that $\left(H M^{\psi}\left(\mathbb{B}_{N}\right)\right)^{* *}$ is the weak-star closure of $H M^{\psi}\left(\mathbb{B}_{N}\right)$ in $L^{\psi}\left(\mathbb{B}_{N}\right)$. Since $H^{\psi}\left(\mathbb{B}_{N}\right)$ is itself weak-star closed, according to 3$)$, the proof will be finished if we show that every function $f$ in $H^{\psi}\left(\mathbb{B}_{N}\right)$ belongs to the weak-star closure of $H M^{\psi}\left(\mathbb{B}_{N}\right)$ in $L^{\psi}\left(\mathbb{S}_{N}\right)$. Now, let $f \in H^{\psi}\left(\mathbb{B}_{N}\right)$; thanks to 1$), f_{r} \underset{r \rightarrow 1}{\longrightarrow} f$ uniformly on every compacta, and therefore (point 2) the convergence occurs for the weak-star topology, for $\left\|f_{r}\right\|_{\psi} \leq\|f\|_{\psi}$ for every $0<r<1$. Therefore $f \in\left(H M^{\psi}\left(\mathbb{B}_{N}\right)\right)^{* *}$ and the proof in complete.

\section{EMbedding THEOREMS FOR HARDY-ORLICZ SPACES}

As usual, our embedding theorems will involve some geometric conditions. The nonisotropic distance on the sphere $\mathbb{S}_{N}$, which we denote by $d$, is given by $d(\zeta, \xi)=\sqrt{|1-\langle\zeta, \xi\rangle|}$, for $(\zeta, \xi) \in \mathbb{S}_{N}$. It can be extended to $\overline{\mathbb{B}_{N}}$, where it still satisfies the triangle inequality (e.g. [15, Paragraph 5.1]). For $\zeta \in \overline{\mathbb{B}_{N}}$ and $\left.\left.h \in\right] 0,1\right]$, we define the non-isotropic "ball" of $\mathbb{B}_{N}$ by

$$
S(\zeta, h)=\left\{z \in \mathbb{B}_{N}, d(\zeta, z)^{2}<h\right\} .
$$

and its analogue in $\overline{\mathbb{B}_{N}}$ by

$$
\mathcal{S}(\zeta, h)=\left\{z \in \overline{\mathbb{B}_{N}}, d(\zeta, z)^{2}<h\right\} .
$$

Let us also denote by

$$
Q=\mathcal{S}(\zeta, h) \cap \mathbb{S}_{N}
$$

the "true" balls in $\mathbb{S}_{N}$. Next, for $\zeta \in \mathbb{S}_{N}$ and $\left.\left.h \in\right] 0,1\right]$, we define the so-called Carleson windows

$$
W(\zeta, h)=\left\{z \in \mathbb{B}_{N}, 1-|z|<h, \frac{z}{|z|} \in Q(\zeta, h)\right\}
$$

and

$$
\mathcal{W}(\zeta, h)=\left\{z \in \overline{\mathbb{B}_{N}}, 1-|z|<h, \frac{z}{|z|} \in Q_{f}(\zeta, h)\right\}
$$

its analogue in $\overline{\mathbb{B}_{N}}$. Finally, we introduce the Korányi approach region $D(\eta)$ for $\eta \in \mathbb{S}_{N}$ by

$$
D(\eta)=\left\{z \in \mathbb{B}_{N}, d(z, \eta)^{2}<1-|z|^{2}\right\} .
$$

Given $f$ continuous on $\mathbb{B}_{N}$ and $\xi \in \mathbb{S}_{N}$, the maximal function $N_{f}$ of $f$ associated to the Korányi approach regions is defined as follows:

$$
N_{f}(\xi)=\sup _{w \in D(\xi)}\{|f(w)|\} .
$$

The Hardy-Littlewood maximal function $M_{f}$ of $f \in L^{1}\left(\mathbb{S}_{N}\right)$ is given by

$$
M_{f}(\xi)=\sup _{\delta>0} \frac{1}{\sigma(Q(\xi, \delta))} \int_{Q(\xi, \delta)}|f| d \sigma_{N},
$$


for $\xi \in \mathbb{S}_{N}$. It is well-known that the sub-linear maximal operator $M: f \longmapsto M_{f}$ is of weak type $(1,1)$ on $\mathbb{S}_{N}$ (see [17], Lemma 4.8) and that it maps $L^{\infty}\left(\mathbb{S}_{N}\right)$ into itself boundedly. Therefore, Proposition 1.2 yields the following result concerning Hardy-Littlewood maximal function on Hardy-Orlicz spaces, under the $\nabla_{2}$-condition:

Theorem 2.1. Let $\psi$ be an Orlicz function satisfying the $\nabla_{2}$-condition. Then, the HardyLittlewood maximal operator $M$ maps $L^{\psi}\left(\mathbb{S}_{N}\right)$ into itself boundedly. More precisely, there exists a constant $C_{\psi}>0$ such that

$$
\left\|M_{f}\right\|_{\psi} \leq C_{\psi}\|f\|_{\psi}
$$

for every $f \in L^{\psi}\left(\mathbb{S}_{N}\right)$.

Moreover, $N_{f}$ is dominated by $M_{f}$ in the "Hardy-Orlicz following sense":

Theorem 2.2. Let $\psi$ be an Orlicz function. Then there exists a constant $C>0$ such that

$$
N_{f}(\xi) \leq C M_{f^{*}}(\xi)
$$

for every $f \in H^{\psi}\left(\mathbb{B}_{N}\right)$ and for every $\xi \in \mathbb{S}_{N}$, where $f^{*} \in L^{\psi}\left(\mathbb{S}_{N}\right)$ is the radial limit of $f$.

Proof. First, $f^{*} \in L^{1}\left(\mathbb{S}_{N}\right)$ since $f \in H^{\psi}\left(\mathbb{B}_{N}\right)$, so $f^{*} d \sigma_{N}$ is a finite complex Borel measure on $\mathbb{S}_{N}$ and we can use [17, Theorem 4.10] to get $N_{P\left[f^{*}\right]} \leq C M_{f^{*}}$ for some constant $C>0$, where $P\left[f^{*}\right](\xi)=\int_{\mathbb{S}_{N}} P(\xi, z) f^{*}(z) d \sigma_{N}(z)$. We finish the proof by noticing that $P\left[f^{*}\right]=f$, for instance because $f \in H^{1}\left(\mathbb{B}_{N}\right)$.

We get a result similar to Theorem 2.1 for the maximal operator $N$ associated to Korányi approach regions:

Corollary 2.3. Let $\psi$ be an Orlicz function satisfying the $\nabla_{2}$-condition. Then, the maximal operator $N$ associated to Korányi approach regions maps $H^{\psi}\left(\mathbb{B}_{N}\right)$ into $L^{\psi}\left(\mathbb{S}_{N}\right)$ boundedly. More precisely, there exists a constant $C_{\psi}>0$ such that

$$
\left\|N_{f}\right\|_{L^{\psi}\left(\mathbb{S}_{N}\right)} \leq C_{\psi}\|f\|_{H^{\psi}\left(\mathbb{B}_{N}\right)},
$$

for every $f \in H^{\psi}\left(\mathbb{B}_{N}\right)$.

Proof. It is an immediate consequence of Theorem 2.1 and Theorem 2.2, using the fact that $\left\|f^{*}\right\|_{L^{\psi}\left(\mathbb{S}_{N}\right)}=\|f\|_{H^{\psi}\left(\mathbb{B}_{N}\right)}$ for every $f \in H^{\psi}\left(\mathbb{B}_{N}\right)$.

For any positive finite Borel measure $\mu$ on $\overline{\mathbb{B}_{N}}$, we introduce the two following functions $\varrho_{\mu}$ and $K_{\mu}$ :

$$
\varrho_{\mu}(h)=\sup _{\xi \in \mathbb{S}_{N}} \mu(\mathcal{W}(\xi, h)) \text { and } K_{\mu}(h)=\sup _{0<t<h} \frac{\mu(\mathcal{W}(\xi, t))}{t^{N}}, \text { for } h \in(0,1) .
$$

We recall that $\mu$ is a Carleson measure if $K_{\mu}(h)$ is finite for some $h \in(0,1)$.

In the sequel, we will assume that the restrictions to $\mathbb{S}_{N}$ of all the measures $\mu$ on $\overline{\mathbb{B}_{N}}$ that we will consider are absolutely continuous with respect to the Lebesgue measure on the sphere.

Theorem 2.4. There exist two constants $\tilde{C}>0$ and $C>1$ such that, for every $f \in$ $H^{1}\left(\mathbb{B}_{N}\right)$ and every positive finite Borel measure $\mu$ on $\overline{\mathbb{B}_{N}}$, we have

$$
\mu\left(\left\{z \in \overline{\mathbb{B}_{N}},|z|>1-h \text { and }|f(z)|>t\right\}\right) \leq \tilde{C} K_{\mu}(C h) \sigma_{N}\left(\left\{N_{f}>t\right\}\right)
$$

for every $h \in(0,1 / C)$ and every $t>0$.

For the proof of this theorem, we will need a covering lemma: 
Lemma 2.5. Let $g$ be a continuous function on $\mathbb{B}_{N}, a>0$ and $h \in(0,1)$. Then, either $|g(w)|<a$ in $\mathbb{B}_{N} \backslash(1-h) \mathbb{B}_{N}$ or there exist $w_{1}, w_{2}, \ldots$ in $\mathbb{B}_{N} \backslash(1-h) \mathbb{B}_{N}$ such that:

(1) $\left|g\left(w_{i}\right)\right| \geq$ a for every $i \geq 1$;

(2) the following inclusion holds:

$$
\left\{w \in \overline{\mathbb{B}_{N}},|g(w)| \geq a\right\} \cap\left(\mathbb{B}_{N} \backslash(1-h) \mathbb{B}_{N}\right) \subset \bigcup_{i \geq 1} \overline{S\left(w_{i}, 4\left(1-\left|w_{i}\right|^{2}\right)\right)}
$$

(3) the sets $Q\left(w_{i},\left(1-\left|w_{i}\right|^{2}\right)\right), i \geq 1$, are pairwise disjoints.

Proof. This lemma is stated in a slightly different form and for $h=1 / 2$ in [13]. There is no difficulty to extend it in the above form and for any $h \in(0,1)$.

Proof of Theorem 2.4. We fix $t>0$. We may suppose that there exists $a \in \mathbb{B}_{N} \backslash(1-h) \mathbb{B}_{N}$ such that $|f(a)|>t$, with $|a|>1-h$. Then the previous lemma ensures that there exists $\left(w_{i}\right)_{i \geq 1} \subset \mathbb{B}_{N} \backslash(1-h) \mathbb{B}_{N}$ such that

$$
\mu\left(\left\{z \in \overline{\mathbb{B}_{N}},|z|>1-h \text { and }|f(z)|>t\right\}\right) \leq \sum_{i \geq 1} \mu\left(\overline{\mathcal{S}\left(w_{i}, 4\left(1-\left|w_{i}\right|^{2}\right)\right)}\right) .
$$

Moreover, because of the definition of the Korányi approach region $D(\eta), \eta \in \mathbb{S}_{N}$, and that of $Q$, we may verify that $N_{f}(\eta) \geq t$ whenever $\eta \in Q\left(w_{i}, 1-\left|w_{i}\right|^{2}\right)$. Therefore, since the sets $Q\left(w_{i}, 1-\left|w_{i}\right|^{2}\right)$ are pairwise disjoints, we have

$$
\sum_{i \geq 1} \sigma_{N}\left(Q\left(w_{i}, 1-\left|w_{i}\right|^{2}\right)\right) \leq \sigma_{N}\left(\left\{N_{f} \geq t\right\}\right)
$$

Now, the triangle inequality ensures that if we set $r=9\left(1-\left|w_{i}\right|^{2}\right)$, then

$$
\mu\left(\overline{\mathcal{S}\left(w_{i}, 4\left(1-\left|w_{i}\right|^{2}\right)\right)}\right) \leq \mu\left(\mathcal{S}\left(\frac{w_{i}}{\left|w_{i}\right|}, r\right)\right) \leq \mu\left(\mathcal{W}\left(\frac{w_{i}}{\left|w_{i}\right|}, C_{0} r\right)\right)
$$

for some $C_{0}>1$. By definition of $K_{\mu}$ and as $r \leq 2 h$, we can find some absolute constant $C>1$ (in fact, we can take $C=2 C_{0}$ ) such that

$$
\mu\left(\overline{\mathcal{S}\left(w_{i}, 4\left(1-\left|w_{i}\right|^{2}\right)\right)}\right) \leq C_{0}^{N} r^{N} \frac{\mu\left(\mathcal{W}\left(\frac{w_{i}}{\left|w_{i}\right|}, C_{0} r\right)\right)}{C_{0}^{N} r^{N}} \leq C_{0}^{N} r^{N} K_{\mu}(C h) .
$$

Now, by using [17, Lemma 4.6] and by homogeneity of the Lebesgue measure on $\mathbb{S}_{N}$, we get

$$
r^{N} \lesssim \sigma_{N}\left(Q\left(\frac{w_{i}}{\left|w_{i}\right|}, r\right)\right) \lesssim \sigma_{N}\left(Q\left(w_{i}, 1-\left|w_{i}\right|^{2}\right)\right)
$$

Hence, inequalities (2.1), (2.2), (2.3) and (2.4) give that there exist two constants $C>1$ and $\tilde{C}>0$ such that

$$
\mu\left(\left\{z \in \overline{\mathbb{B}_{N}},|z|>1-h \text { and }|f(z)|>t\right\}\right) \leq \tilde{C} K_{\mu}(C h) \sigma_{N}\left(\left\{N_{f} \geq t\right\}\right) .
$$

The next technical lemma is a consequence of Theorem 2.4.

Lemma 2.6. Let $\mu$ be a finite positive Borel measure on $\overline{\mathbb{B}_{N}}$ and let $\psi_{1}$ and $\psi_{2}$ be two Orlicz functions. Let $C \geq 1$ be the constant appearing in Theorem 2.4. Assume that there exist $A>0, \eta>0$ and $h_{A} \in(0,1 / C)$ such that

$$
K_{\mu}(h) \leq \eta \frac{1 / h^{N}}{\psi_{2}\left(A \psi_{1}^{-1}\left(1 / h^{N}\right)\right)}
$$


for every $h \in\left(0, h_{A}\right)$. Then, there exist three constants $B>0, x_{A}>0$ and $C_{1}>0$ (this latter does not depend on $A, \eta$ and $h_{A}$ ) such that, for every $f \in H^{\psi_{1}}\left(\mathbb{B}_{N}\right)$ such that $\|f\|_{\psi_{1}} \leq 1$ and every Borel subset $E$ of $\overline{\mathbb{B}_{N}}$,

$$
\int_{E} \psi_{2}\left(\frac{|f|}{B}\right) d \mu \leq \mu(E) \psi_{2}\left(x_{A}\right)+C_{1} \eta \int_{\mathbb{S}_{N}} \psi_{1}\left(N_{f}\right) d \sigma_{N} .
$$

Proof. For $f \in H^{\psi_{1}}\left(\mathbb{B}_{N}\right),\|f\|_{\psi_{1}} \leq 1$, using a classical Fubini integration argument, we have

$$
\int_{E} \psi_{2}(|f|) d \mu=\int_{0}^{\infty} \psi_{2}^{\prime}(t) \mu(\{|f|>t\} \cap E) d t .
$$

We shall pay attention to $\mu(\{|f|>t\})$. If $|f(z)|>t$, Proposition 1.7 gives

$$
t \leq 2^{N+1} \psi_{1}^{-1}\left(\left(\frac{1}{1-|z|}\right)^{N}\right)
$$

since $\|f\|_{\psi_{1}} \leq 1$, which is clearly equivalent to

$$
|z|>1-\left(\frac{1}{\psi_{1}\left(\frac{t}{2^{N+1}}\right)}\right)^{1 / N}
$$

Theorem 2.4 then yields

$$
\begin{aligned}
\mu(\{|f|>t\}) & =\mu\left(\{|f|>t\} \cap\left\{|z|>1-\left(\frac{1}{\psi_{1}\left(\frac{t}{2^{N+1}}\right)}\right)^{1 / N}\right\}\right) \\
& \leq K_{\mu}\left(C\left(\frac{1}{\psi_{1}\left(\frac{t}{2^{N+1}}\right)}\right)^{1 / N}\right) \sigma_{N}\left(\left\{N_{f}>t\right\}\right) .
\end{aligned}
$$

Now, let $A, \eta, h_{A}$ and $E$ be as in the statement of the lemma. We set

$$
x_{A}:=\frac{A}{(C+1) C^{N-1}} \psi_{1}^{-1}\left(\left(\frac{C}{h_{A}}\right)^{N}\right)
$$

the assumption of the lemma ensures that, if $\frac{1}{C^{N}} \psi_{1}\left(\frac{(C+1) C^{N-1}}{A} s\right)>1 / h_{A}^{N}$ i.e. if $s \geq$ $x_{A}$, then

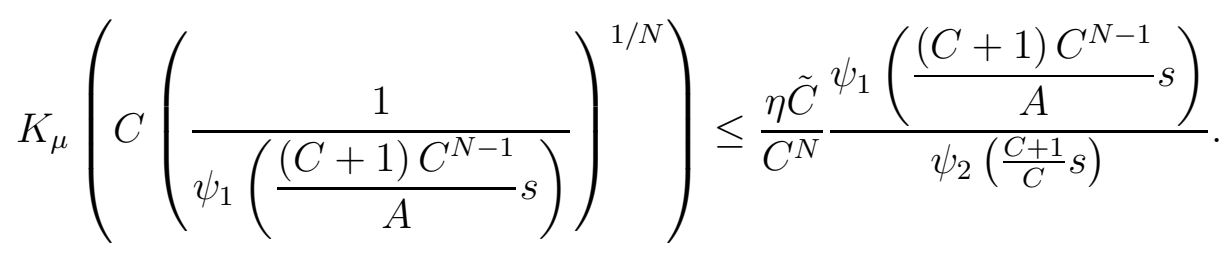


Therefore, applying (2.5) to $\frac{A}{2^{N+1}(C+1) C^{N-1}}|f|$, we deduce from inequalities (2.7) with $t=\frac{2^{N+1}(C+1) C^{N-1} s}{A}$ and (2.8) that

$$
\begin{aligned}
& \int_{E} \psi_{2}\left(\frac{A}{2^{N+1}(C+1) C^{N-1}}|f|\right) d \mu \leq \int_{0}^{x_{A}} \psi_{2}^{\prime}(s) \mu(E) d s \\
& \quad+\frac{\eta \tilde{C}}{C^{N}} \int_{x_{A}}^{\infty} \psi_{2}^{\prime}(s) \frac{\psi_{1}\left(\frac{(C+1) C^{N-1}}{A} s\right)}{\psi_{2}\left(\frac{C+1}{C} s\right)} \sigma_{N}\left(\left\{N_{f}>\frac{2^{N+1}(C+1) C^{N-1}}{A} s\right\}\right) d s .
\end{aligned}
$$

Now, it is not difficult to check that, for any Orlicz function $\psi$, we have

$$
x \psi^{\prime}(x) \leq C \psi\left(\frac{(C+1) x}{C}\right)
$$

for every $C>0$ and every $x \geq 0$, so that

$$
\frac{\psi_{2}^{\prime}(s)}{\psi_{2}\left(\frac{C+1}{C} s\right)} \leq \frac{C}{s}
$$

So inequality (2.9) becomes

$$
\begin{aligned}
& \int_{E} \psi_{2}\left(\frac{A}{2^{N+1}(C+1) C^{N-1}}|f|\right) d \mu \leq \psi_{2}\left(x_{A}\right) \mu(E) \\
+ & \frac{\eta \tilde{C}}{C^{N-1}} \frac{(C+1) C^{N-1}}{A} \int_{0}^{\infty} \psi_{1}^{\prime}\left(\frac{(C+1) C^{N-1}}{A} s\right) \sigma_{N}\left(\left\{N_{f}>\frac{2^{N+1}(C+1) C^{N-1}}{A} s\right\}\right) d s,
\end{aligned}
$$

using the convexity of $\psi_{1}$ one more time. A change of variable gives:

$$
\begin{aligned}
\int_{E} \psi_{2}\left(\frac{A|f|}{2^{N+1} C^{N-1}(C+1)}\right) d \mu & \leq \psi_{2}\left(x_{A}\right) \mu(E)+\frac{\eta \tilde{C}}{C^{N-1}} \int_{0}^{\infty} \psi_{1}^{\prime}(u) \sigma_{N}\left(N_{f}>2^{N+1} u\right) d u \\
& \leq \psi_{2}\left(x_{A}\right) \mu(E)+\frac{\eta \tilde{C}}{2^{N+1} C^{N-1}} \int_{\mathbb{S}_{N}} \psi_{1}\left(N_{f}\right) d \sigma
\end{aligned}
$$

which finishes the proof.

2.1. The canonical embedding $H^{\psi_{1}}\left(\mathbb{B}_{N}\right) \hookrightarrow L^{\psi_{2}}(\mu)$. The main theorem of this section is the following:

Theorem 2.7. Let $\mu$ be a finite positive Borel measure on $\overline{\mathbb{B}_{N}}$ and let $\psi_{1}$ and $\psi_{2}$ be two Orlicz functions; we suppose that $\psi_{1}$ satisfies the $\nabla_{2}$-condition. Then:

(1) If inclusion $H^{\psi_{1}}\left(\mathbb{B}_{N}\right) \subset L^{\psi_{2}}(\mu)$ holds and is continuous, then there exists some $A>0$ such that

$$
\varrho_{\mu}(h)=O_{h \rightarrow 0}\left(\frac{1}{\psi_{2}\left(A \psi_{1}^{-1}\left(1 / h^{N}\right)\right)}\right) .
$$

(2) If there exists some $A>0$ such that

$$
K_{\mu}(h)=O_{h \rightarrow 0}\left(\frac{1 / h^{N}}{\psi_{2}\left(A \psi_{1}^{-1}\left(1 / h^{N}\right)\right)}\right)
$$

then inclusion $H^{\psi_{1}}\left(\mathbb{B}_{N}\right) \subset L^{\psi_{2}}(\mu)$ holds and is continuous. 
(3) If in addition $\psi_{1}=\psi_{2}=\psi$ satisfies the uniform $\nabla_{0}$-Condition, then Conditions (2.10) and (2.11) are equivalent.

Proof. Let us denote by $j_{\mu}$ the embedding $H^{\psi_{1}}\left(\mathbb{B}_{N}\right) \hookrightarrow L^{\psi_{2}}(\mu)$ and $C_{\mu}$ its norm (possibly infinite). Note that $C_{\mu}$ is finite as soon as $j_{\mu}$ is well-defined, because of the closed graph theorem.

1) We assume that $j_{\mu}$ is well-defined (or equivalently that it is bounded). For $a \in \mathbb{S}_{N}$ and $h \in(0,1)$, we define

$$
f_{a, h}=\psi^{-1}\left(\frac{1}{h^{N}}\right) u_{a, 1-h}
$$

where $u_{a, 1-h}(z)=\left(\frac{h}{1-(1-h)\langle z, a\rangle}\right)^{2 N}$. As we saw in the previous section, $f$ lays in the unit ball of $H^{\psi_{1}}\left(\mathbb{B}_{N}\right)$, so that $\left\|j_{\mu}\left(f_{a, h}\right)\right\|_{L^{\psi_{2}(\mu)}}=\left\|f_{a, h}\right\|_{L^{\psi_{2}(\mu)}}$ must be not greater than $C_{\mu}$. It follows that $\int_{\overline{\mathbb{B}_{N}}} \psi_{2}\left(|f| / C_{\mu}\right) d \mu \leq 1$. Now, we may easily check that $|1-(1-h)\langle z, a\rangle| \leq 2 h$ whenever $z \in \mathcal{S}(a, h)$, hence $\left|u_{a, 1-h}(z)\right| \geq \frac{1}{4^{N}}$ and $|f(z)| \geq \frac{1}{4^{N}} \psi_{1}^{-1}\left(\frac{1}{h^{N}}\right)$ for any $z \in$ $\mathcal{S}(a, h)$. Consequently, integrating on $\mathcal{S}(a, h)$, we get

$$
1 \geq \int_{\mathcal{S}(a, h)} \psi_{2}\left(\frac{1}{4^{N}} \psi_{1}^{-1}\left(\frac{1}{h^{N}}\right)\right) d \mu \geq \psi_{2}\left(\frac{1}{4^{N}} \psi_{1}^{-1}\left(\frac{1}{h^{N}}\right)\right) \mu(\mathcal{S}(a, h)),
$$

which yields Condition (2.10) and the first part of the theorem.

2) This part need Lemma 2.6. Since $\psi_{1}$ satisfies $\nabla_{2}$-Condition, Corollary 2.3 ensures that there exists some constant $C_{M} \geq 1$ such that, for any $f \in L^{\psi_{1}}\left(\mathbb{S}_{N}\right),\left\|N_{f}\right\|_{L^{\psi_{1}\left(\mathbb{S}_{N}\right)}} \leq$ $C_{M}\|f\|_{H^{\psi_{1}\left(\mathbb{B}_{N}\right)}}$. We fix $f$ in the unit ball of $H^{\psi_{1}}\left(\mathbb{B}_{N}\right)$ and we intend to show that $\|f\|_{L^{\psi_{2}(\mu)}} \leq$ $C_{0}$ for some $C_{0}>0$ independent of $f$. We also introduce a constant $\tilde{C} \geq 1$ whose value will be fixed later.

Now, since Condition (2.10) is supposed to be realized, we shall apply Lemma 2.6 to $f / C_{M}$, with $E=\overline{\mathbb{B}_{N}}, \eta$ and $h_{A}$, and we get the existence of $B>0, x_{A}>0$ and $C_{1}>0$, all independent of $f$ such that

$$
\begin{aligned}
\int_{\mathbb{B}_{N}} \psi_{2}\left(\frac{|f|}{B C_{M} \tilde{C}}\right) d \mu & \leq \frac{1}{\tilde{C}} \int_{\overline{\mathbb{B}_{N}}} \psi_{2}\left(\frac{|f|}{B C_{M}}\right) d \mu \\
& \leq \frac{1}{\tilde{C}}\left(\mu\left(\overline{\mathbb{B}_{N}}\right) \psi_{2}\left(x_{A}\right)+C_{1} \eta \int_{\mathbb{S}_{N}} \psi_{1}\left(\frac{1}{C_{M}} N_{f}\right) d \sigma_{N}\right) \\
& \leq \frac{1}{\tilde{C}}\left(\mu\left(\overline{\mathbb{B}_{N}}\right) \psi_{2}\left(x_{A}\right)+C_{1} \eta\right) .
\end{aligned}
$$

Of course, $C_{1}$ may be chosen so that $C_{1} \eta \geq 1$ and putting $\tilde{C}=\mu\left(\overline{\mathbb{B}_{N}}\right) \psi_{2}\left(x_{A}\right)+C_{1} \eta \geq 1$, we get $\|f\|_{L^{\psi_{2}(\mu)}} \leq C_{0}:=B C_{M} \tilde{C}$, hence the second part of Theorem 2.7.

3) First, it is clear that Condition (2.11) implies Condition (2.10). The converse is based on the following claim:

Claim. If Condition (2.10) is satisfied, then there exist some $A$ as large as we want and $\eta>0$ such that

$$
\varrho_{\mu}(h) \leq \eta \frac{1}{\psi_{2}\left(A \psi_{1}^{-1}\left(h_{A} / h^{N}\right)\right)}
$$

for some $h_{A}, 0<h_{A} \leq 1$ and for any $0<h<h_{A}$. 
Proof of the claim. We assume that Condition

$$
\varrho_{\mu}(h) \leq \eta \frac{1}{\psi_{2}\left(\tilde{A} \psi_{1}^{-1}\left(1 / h^{N}\right)\right)}
$$

holds for some $\tilde{A} \geq 0, \tilde{h_{A}}, 0<\tilde{h_{A}} \leq 1, \eta>0$ and any $0<h<\tilde{h_{A}}$. We fix $A>1$ and we look for some constant $h_{\tilde{A}, A} \leq 1$ such that

$$
\frac{1}{\psi_{2}\left(\tilde{A} \psi_{1}^{-1}\left(1 / h^{N}\right)\right)} \leq \frac{1}{\psi_{2}\left(A \psi_{1}^{-1}\left(\left(h_{\tilde{A}, A} / h\right)^{N}\right)\right)}
$$

for $0<h<h_{\tilde{A}, A}$. Now it is easy to verify that Inequality (2.14) is equivalent to

$$
\frac{A}{\tilde{A}} \leq \frac{\psi_{1}^{-1}\left(1 / h^{N}\right)}{\psi_{1}^{-1}\left(\left(h_{\tilde{A}, A} / h\right)^{N}\right)} \leq \frac{1}{h_{\tilde{A}, A}^{N}}
$$

by concavity of $\psi_{1}^{-1}$. Then the claim follows by choosing $h_{\tilde{A}, A}$ small enough.

We finish the proof of the third point of the theorem. Let assume that $\psi_{1}=\psi_{2}=\psi$ belongs to the uniform $\nabla_{0}$-Class and that Condition (2.10) is satisfied for some constant $A>0$. The previous claim says that we can find $B \geq 1$ and $0<K=K_{B, A} \leq 1$ such that

$$
\varrho_{\mu}(h) \leq \eta \frac{1}{\psi\left(B \psi^{-1}\left((K / h)^{N}\right)\right)}
$$

for every $0<h<K$. Therefore, we have

$$
K_{\mu}(h)=\sup _{0<t \leq h} \frac{\varrho_{\mu}(t)}{t^{N}} \leq \eta \sup _{0<t \leq h} \frac{1 / t^{N}}{\psi\left(B \psi^{-1}\left((K / t)^{N}\right)\right)}=\eta \sup _{x \geq \psi^{-1}\left((K / h)^{N}\right)} \frac{1}{K^{N}} \frac{\psi(x)}{\psi(B x)},
$$

for any $0<h \leq K$. Let $C$ be the constant induced by the uniform $\nabla_{0}$-Condition satisfied by $\psi$ and let $\beta$ be such that $B=\beta C$. According to the claim, $B$ can be chosen large enough so that $\beta>1$. Then, using uniform $\nabla_{0^{-}}$Condition, we get

$$
\frac{\psi\left(\beta \psi^{-1}\left((K / h)^{N}\right)\right)}{(K / h)^{N}} \leq \frac{\psi(B x)}{\psi(x)}
$$

for any $x \geq \psi^{-1}\left((K / h)^{N}\right)$. Hence, for every $0<h \leq K$,

$$
K_{\mu}(h) \leq \eta \frac{1 / h^{N}}{\psi\left(\beta \psi^{-1}\left((K / h)^{N}\right)\right)} \leq \eta \frac{1 / h^{N}}{\psi\left(\beta K^{N} \psi^{-1}\left(1 / h^{N}\right)\right)}
$$

by concavity of $\psi^{-1}$, which is (2.11).

The previous theorem leads us to introduce the $\psi$-Carleson measures:

Definition 2.8. Let $\mu$ be a finite positive Borel measure on $\overline{\mathbb{B}_{N}}$ and let $\psi$ be an Orlicz function. We say that $\mu$ is a $\psi$-Carleson measure if there exists $A>0$, such that

$$
\mu(\mathcal{S}(\xi, h))=O_{h \rightarrow 0}\left(\frac{1}{\psi\left(A \psi^{-1}\left(1 / h^{N}\right)\right)}\right)
$$

uniformly with respect to $\xi \in \mathbb{S}_{N}$.

We remark that (2.15) is equivalent to (2.10), and we have the following corollary, by noticing that the uniform $\nabla_{0}$-Condition implies the $\nabla_{2}$-Condition (Proposition 1.5): 
Corollary 2.9. Let $\mu$ be a finite positive Borel measure on $\overline{\mathbb{B}_{N}}$ and let $\psi$ be an Orlicz function. Then, if $\psi$ satisfies the uniform $\nabla_{0}$-Condition, then inclusion $H^{\psi}\left(\mathbb{B}_{N}\right) \subset L^{\psi}(\mu)$ holds (and is continuous) if and only if $\mu$ is a $\psi$-Carleson measure.

2.2. Compactness of the canonical embedding $H^{\psi_{1}}\left(\mathbb{B}_{N}\right) \hookrightarrow L^{\psi_{2}}(\mu)$. For this purpose, we need a criterion of compactness for embedding operators from $H^{\psi_{1}}\left(\mathbb{B}_{N}\right)$ into $L^{\psi_{2}}(\mu)$. First of all, we shall state the following proposition:

Proposition 2.10. Let $\mu$ be a finite positive Borel measure on $\overline{\mathbb{B}_{N}}$ (whose restriction to $\mathbb{S}_{N}$ is absolutely continuous with respect to $\left.\sigma_{N}\right)$; let $\psi_{1}$ and $\psi_{2}$ be two Orlicz functions. If the canonical embedding $H^{\psi_{1}}\left(\mathbb{B}_{N}\right) \hookrightarrow L^{\psi_{2}}(\mu)$ is compact, then $\mu\left(\mathbb{S}_{N}\right)=0$.

Proof. We assume that $j_{\mu}: H^{\psi_{1}}\left(\mathbb{B}_{N}\right) \hookrightarrow L^{\psi_{2}}(\mu)$ is compact. Let $f: \mathbb{B}_{N} \rightarrow \mathbb{C}$ be an inner function ([1]); the sequence $\left(f^{n}\right)_{n}$ lays in the unit ball of $H^{\psi_{1}}\left(\mathbb{B}_{N}\right)$ hence, by compactness of $j_{\mu}$ and up to an extraction of a subsequence, we may suppose that $\left(j_{\mu}\left(f^{n}\right)\right)_{n}=\left(f^{n}\right)_{n}$ is a Cauchy sequence in $L^{1}(\mu)$, for $L^{\psi_{2}}(\mu) \subset L^{1}(\mu)$. Now, since $\mu_{\mid \mathbb{S}_{N}}$ is absolutely continuous with respect to the Lebesgue measure $\sigma_{N}$, and since $f$ is inner, we must have

$$
\left\|f^{n}-f^{m}\right\|_{L^{1}\left(\mu_{\mid \mathbb{S}_{N}}\right)}=\left\|1-f^{m-n}\right\|_{L^{1}\left(\mu_{\mid S_{N}}\right)} \underset{n, m \rightarrow \infty}{\longrightarrow} 0 .
$$

By contradiction, we assume that $\mu_{\mid \mathbb{S}_{N}}>0$. We can then extract a subsequence $\left(f^{n_{k}}\right)_{n_{k}}$ which converges to $1 \mu_{\mid \mathbb{S}_{N}}$-a.e. and by Egoroff's theorem, the convergence is uniform on a set $E \subset \mathbb{S}_{N}$ of measure $\mu_{\mid \mathbb{S}_{N}}$ positive. Because $\mu_{\mid \mathbb{S}_{N}}$ is absolutely continuous with respect to $\sigma_{N}$, we must have $\sigma_{N}(E)>0$. Now, by subharmonicity of $\log \left|1-f^{n_{k}}\right|$, it follows that

$$
\log \left|1-f^{n_{k}}(0)\right| \leq \int_{E} \log \left|1-f^{n_{k}}\right| d \sigma_{N}+\int_{\mathbb{S}_{N} \backslash E} \log \left|1-f^{n_{k}}\right| d \sigma_{N} .
$$

The right hand side of this inequality now tends to $-\infty$ as $k \rightarrow \infty$, because $\log \left|1-f^{n_{k}}\right|$ is uniformly convergent to $-\infty$ on $E$ and $\log \left|1-f^{n_{k}}\right| \leq \log 2$ a.e. We get a contradiction, for $f^{n_{k}}(0)$ tends to 0 as $k \rightarrow \infty$.

We give a necessary and sufficient condition for the canonical embedding $H^{\psi_{1}}\left(\mathbb{B}_{N}\right) \hookrightarrow$ $L^{\psi_{2}}(\mu)$ to be compact.

Proposition 2.11. Let $\mu$ be a finite positive measure on $\overline{\mathbb{B}_{N}}$ and let $\psi_{1}$ and $\psi_{2}$ be two Orlicz functions. We suppose that the canonical embedding $j_{\mu}: H^{\psi_{1}}\left(\mathbb{B}_{N}\right) \hookrightarrow L^{\psi_{2}}(\mu)$ holds (and is bounded).

(1) The two following assertions are equivalent:

(a) The canonical embedding $H^{\psi_{1}}\left(\mathbb{B}_{N}\right) \hookrightarrow L^{\psi_{2}}(\mu)$ is compact;

(b) Every sequence in the unit ball of $H^{\psi_{1}}\left(\mathbb{B}_{N}\right)$, which is convergent to 0 uniformly on every compact subset of $\mathbb{B}_{N}$, is convergent to 0 in $L^{\psi_{2}}(\mu)$.

(2) If $H^{\psi_{1}}\left(\mathbb{B}_{N}\right)$ is continuously embedded in $L^{\psi_{2}}(\mu)$ and if $\lim _{r \rightarrow 1^{-}}\left\|I_{r}\right\|=0$, where $I_{r}(f)=f \cdot \chi_{\mathbb{B}_{N}} \backslash r \mathbb{B}_{N}$, then the canonical embedding $H^{\psi_{1}}\left(\mathbb{B}_{N}\right) \hookrightarrow L^{\psi_{2}}(\mu)$ is compact.

Proof. 1) (a) $\Rightarrow$ (b) Let $\left(f_{n}\right)_{n}$ be a sequence in the unit ball of $H^{\psi_{1}}\left(\mathbb{B}_{N}\right)$, which converges to 0 uniformly on every compact subset of $\mathbb{B}_{N}$. Since $j_{\mu}$ is compact, $\mu_{\mid \mathbb{S}_{N}}=0$ according to Proposition 2.10, so that $f_{n} \underset{n \rightarrow \infty}{\longrightarrow} 0 \mu$-a.e. By contradiction, suppose up to extract a subsequence that $\liminf _{n}\left\|f_{n}\right\|_{L^{\psi_{2}(\mu)}}>0$. By compactness of $j_{\mu}$, up to an other extraction, we may assume that $\left(f_{n}\right)_{n}$ converges to some $g$ in $L^{\psi_{2}}(\mu)$, which must satisfy $\|g\|_{L^{\psi_{2}(\mu)}}>0$. Then, $f_{n} \underset{n \rightarrow \infty}{\longrightarrow} g \mu$-a.e and we get a contradiction.

(b) $\Rightarrow$ (a) Conversely, let $\left(f_{n}\right)_{n}$ be a sequence in the unit ball of $H^{\psi_{1}}\left(\mathbb{B}_{N}\right)$. In particular, $\left(f_{n}\right)_{n}$ is in the unit ball of $H^{1}\left(\mathbb{B}_{N}\right)$ and Cauchy's formula ensures that $\left(f_{n}\right)_{n}$ is uniformly 
bounded on every compact subset of $\mathbb{B}_{N}$, so that, up to an extraction, we may suppose that $\left(f_{n}\right)_{n}$ is uniformly convergent on compact subsets of $\mathbb{B}_{N}$ to $f$ holomorphic in $\mathbb{B}_{N}$, by Montel's theorem. Now, Lebesgue's theorem ensures that $f \in H^{\psi_{1}}\left(\mathbb{B}_{N}\right)$ and, up to divide by a constant large enough, we may assume that $f_{n}-f$, which converges to 0 on every compact subset of $\mathbb{B}_{N}$, lays in the unit ball of $H^{\psi_{1}}\left(\mathbb{B}_{N}\right)$. Therefore, our assumption implies that $\left(j_{\mu}\left(f_{n}\right)-j_{\mu}(f)\right)_{n}$ converges to 0 in the norm of $L^{\psi_{2}}(\mu)$ and $j_{\mu}$ is compact, as expected.

2) Thanks to 1), it is sufficient to show that if $\lim _{r \rightarrow 1^{-}}\left\|I_{r}\right\|=0$, then (b) is satisfied. Let $\left(f_{n}\right)_{n}$ be in the unit ball of $H^{\psi_{1}}\left(\mathbb{B}_{N}\right)$ converging to 0 uniformly on every compact subset of $\mathbb{B}_{N}$. We have

$$
\begin{aligned}
& \limsup _{n \rightarrow \infty}\left\|f_{n}\right\|_{L^{\psi_{2}(\mu)}}=\limsup _{r \rightarrow 1^{-}} \limsup _{n \rightarrow \infty}\left\|I_{r}\left(f_{n}\right)+f_{n} \cdot \chi_{r \mathbb{B}_{N}}\right\|_{L^{\psi_{2}(\mu)}} \\
& \lesssim \limsup _{r \rightarrow 1^{-}}\left\|I_{r}\right\|+\limsup _{r \rightarrow 1^{-}} \limsup _{n \rightarrow \infty}\left\|f_{n} \cdot \chi_{r \mathbb{B}_{N}}\right\|_{\infty} \\
& =0 \text {. }
\end{aligned}
$$

We shall now state and prove our main theorem about compactness of the canonical embedding $H^{\psi_{1}}\left(\mathbb{B}_{N}\right) \hookrightarrow L^{\psi_{2}}(\mu)$.

Theorem 2.12. Let $\mu$ be a finite positive Borel measure on $\overline{\mathbb{B}_{N}}$, and let $\psi_{1}$ and $\psi_{2}$ be two Orlicz functions. We assume that $\psi_{1}$ satisfies the $\nabla_{2}$-condition. Then:

(1) If inclusion $H^{\psi_{1}}\left(\mathbb{B}_{N}\right) \subset L^{\psi_{2}}(\mu)$ holds and is compact, then for every $A>0$ we have

$$
\varrho_{\mu}(h)=o_{h \rightarrow 0}\left(\frac{1}{\psi_{2}\left(A \psi_{1}^{-1}\left(1 / h^{N}\right)\right)}\right) .
$$

(2) If

$$
K_{\mu}(h)=o_{h \rightarrow 0}\left(\frac{1 / h^{N}}{\psi_{2}\left(A \psi_{1}^{-1}\left(1 / h^{N}\right)\right)}\right)
$$

for every $A>0$, then inclusion $H^{\psi_{1}}\left(\mathbb{B}_{N}\right) \subset L^{\psi_{2}}(\mu)$ holds and is compact.

(3) If in addition $\psi_{1}=\psi_{2}=\psi$ satisfies both $\nabla_{0}$ and $\nabla_{2}$-Conditions then Conditions (2.16) and (2.17) are equivalent.

Proof. 1) By contradiction, we assume that $H^{\psi_{1}}\left(\mathbb{B}_{N}\right) \subset L^{\psi_{2}}(\mu)$ is compact, whereas Condition (2.16) is not satisfied: there exist $\varepsilon_{0} \in(0,1), A>0$, a sequence $\left(h_{n}\right)_{n} \subset(0,1)$ decreasing to 0 , and a sequence $\xi_{n} \subset \mathbb{S}_{N}$, such that

$$
\mu\left(\mathcal{S}\left(\xi_{n}, h_{n}\right)\right) \geq \frac{\varepsilon_{0}}{\psi_{2}\left(A \psi_{1}^{-1}\left(1 / h^{N}\right)\right)},
$$

for any $n \in \mathbb{N}$. We consider the test functions introduced in the proof of Theorem 2.7:

$$
f_{n}=\psi_{1}^{-1}\left(1 / h_{n}^{N}\right) u_{\xi_{n}, 1-h_{n}} .
$$

Each $f_{n}$ lays in the unit ball of $H^{\psi_{1}}\left(\mathbb{B}_{N}\right)$ and $\left(f_{n}\right)_{n}$ tends to 0 uniformly on every compacta of $\mathbb{B}_{N}$ so that Proposition 2.11 ensures that $\left(f_{n}\right)_{n}$ tends to 0 in $L^{\psi_{2}}(\mu)$. Now, we showed in Theorem 2.7 that

$$
|f(z)| \geq \frac{1}{4^{N}} \psi_{1}^{-1}\left(\frac{1}{h^{N}}\right)
$$


for any $z \in \mathcal{S}\left(\xi_{n}, h_{n}\right)$. Hence

$$
\begin{aligned}
\int_{\overline{\mathbb{B}_{N}}} \psi_{2}\left(\frac{4^{N} A}{\varepsilon_{0}}\left|f_{n}\right|\right) d \mu & \geq \psi_{2}\left(\frac{A}{\varepsilon_{0}} \psi_{1}^{-1}\left(\frac{1}{h^{N}}\right)\right) \mu\left(\mathcal{S}\left(\xi_{n}, h_{n}\right)\right) \\
& \geq \psi_{2}\left(\frac{A}{\varepsilon_{0}} \psi_{1}^{-1}\left(\frac{1}{h^{N}}\right)\right) \frac{\varepsilon_{0}}{\psi_{2}\left(A \psi_{1}^{-1}\left(1 / h^{N}\right)\right)} \\
& \geq 1
\end{aligned}
$$

using convexity of $\psi_{2}$. Therefore, $\left\|f_{n}\right\|_{L^{\psi_{2}(\mu)}} \geq \frac{\varepsilon_{0}}{4^{N} A}$ for any $n$, which contradicts the fact that $\left\|f_{n}\right\|_{L^{\psi_{2}(\mu)}}$ tends to 0 .

2) We assume that Condition (2.17) is satisfied. Theorem 2.7 ensures that inclusion $H^{\psi_{1}}\left(\mathbb{B}_{N}\right) \subset L^{\psi_{2}}(\mu)$ holds (and is bounded). Thanks to Proposition 2.11, it is sufficient to show that, for every $\varepsilon>0$, if we denote by $I_{r}: H^{\psi_{1}}\left(\mathbb{B}_{N}\right) \rightarrow L^{\psi_{2}}\left(\overline{\mathbb{B}_{N}} \backslash r \mathbb{B}_{N}, \mu\right)$ as in the second point of Proposition 2.11, then $\left\|I_{r}\right\|<\varepsilon$ whenever $r$ is closed enough to 1. Let $\eta \in(0,1)$ and let us set $A=\frac{2^{N+1}(C+1) C^{N-1}}{\varepsilon}>0$, where $C$ is the constant involved in Theorem 2.4, Condition (2.17) yields the existence of a constant $h_{A} \in(0,1 / C)$ such that

$$
K_{\mu}(h) \leq \eta \frac{1 / h^{N}}{\psi_{2}\left(A \psi_{1}^{-1}\left(1 / h^{N}\right)\right)},
$$

for any $h \leq h_{A}$. Now, if $f$ is in the unit ball of $H^{\psi_{1}}\left(\mathbb{B}_{N}\right)$ and if $r \in(0,1)$ is given, Lemma 2.6. applied to $E=\overline{\mathbb{B}_{N}} \backslash r \mathbb{B}_{N}$ and to $f$, provides a constant $B>0$, given by $B=\frac{2^{N+1}(C+1) C^{N-1}}{A}=\varepsilon$, and $x_{A}>0$ and $C_{1}>0$ independent of $f$, such that

$$
\begin{aligned}
\int_{\overline{\mathbb{B}_{N}} \backslash r B_{N}} \psi_{2}\left(\frac{|f|}{\varepsilon}\right) d \mu & =\int_{\overline{\mathbb{B}_{N}} \backslash r B_{N}} \psi_{2}\left(\frac{|f|}{B}\right) d \mu \\
& \leq \mu\left(\overline{\mathbb{B}_{N}} \backslash r \mathbb{B}_{N}\right) \psi_{2}\left(x_{A}\right)+C_{1} \eta \int_{\mathbb{S}_{N}} \psi_{1}\left(N_{f}\right) d \sigma_{N} .
\end{aligned}
$$

Furthermore, $\eta>0$ is chosen in order that $C_{1} \eta \int_{\mathbb{S}_{N}} \psi_{1}\left(N_{f}\right) d \sigma_{N} \leq \frac{1}{2}$ (which is possible thanks to Corollary 2.3 , since $\psi_{1}$ satisfies the $\nabla_{2}$-Condition). For the end of the proof, we need a lemma:

Lemma 2.13. Under the assumptions of Theorem 2.12, if Condition (2.17) is satisfied, then $\mu\left(\mathbb{S}_{N}\right)=0$.

Proof. Thanks to [15, Lemma 5.2.3], for any $h \in(0,1)$, if $C(h)$ denotes the minimal number of non-isotropic balls $Q(\zeta, h)$ which are needed to cover $\mathbb{S}_{N}$, then there exists $C>0$, independent of $h$, such that $C(h) \leq \frac{C}{h^{N}}$. Therefore, if Condition (2.17) holds, then

$$
\mu\left(\overline{\mathbb{B}_{N}} \backslash(1-h) \mathbb{B}_{N}\right) \leq C \frac{\varrho_{\mu}(h)}{h^{N}} \leq C K_{\mu}(h) \underset{h \rightarrow 0}{\longrightarrow} 0 .
$$

We finish the proof of Theorem 2.12 by considering some $r_{0} \in(0,1)$ such that

$$
\mu\left(\overline{\mathbb{B}_{N}} \backslash r \mathbb{B}_{N}\right) \psi_{2}\left(x_{A}\right) \leq \frac{1}{2}
$$

for any $r, r_{0}<r<1$, thanks to $\mu\left(\mathbb{S}_{N}\right)=0$. We deduce that $\left\|I_{r}(f)\right\|_{L^{\psi_{2}(\mu)}} \leq \varepsilon$ in (2.18), for each $r>r_{0}$, which ends the proof. 
3) This is quite similar to $[9$, Theorem 4.11,3)].

The third point of the theorem leads us to define what one calls vanishing $\psi$-Carleson measures:

Definition 2.14. Let $\mu$ be a finite positive Borel measure on $\overline{\mathbb{B}_{N}}$ and let $\psi$ be an Orlicz function. We say that $\mu$ is a vanishing $\psi$-Carleson measure if, for every $A>0$,

$$
\mu(\mathcal{S}(\xi, h))=o_{h \rightarrow 0}\left(\frac{1}{\psi\left(A \psi^{-1}\left(1 / h^{N}\right)\right)}\right)
$$

uniformly with respect to $\xi \in \mathbb{S}_{N}$.

We now state the following corollary:

Corollary 2.15. Let $\psi$ be an Orlicz function and let $\mu$ be a finite positive Borel measure on $\overline{\mathbb{B}_{N}}$. If $\psi$ satisfies the $\nabla_{0} \cap \nabla_{2}$-Condition, then the embedding $H^{\psi}\left(\mathbb{B}_{N}\right) \hookrightarrow L^{\psi}(\mu)$ holds and is compact if and only if $\mu$ is a vanishing $\psi$-Carleson measure.

\section{Application to composition operators on Hardy-Orlicz spaces}

In this section, we denote by $\phi: \mathbb{B}_{N} \rightarrow \mathbb{B}_{N}$ an holomorphic map and by $\phi^{*}: \overline{\mathbb{B}_{N}} \rightarrow \overline{\mathbb{B}_{N}}$ the map which is equal to $\phi$ on $\mathbb{B}_{N}$ and which is equal to the $\sigma_{N^{-}}$a.e. boundary limit of $\phi$ on $\mathbb{S}_{N}$, as usual; we define the pull-back measure $\mu_{\phi}$ on $\overline{\mathbb{B}_{N}}$ induced by $\phi^{*}$ as the image of the normalized Lebesgue measure $\sigma_{N}$ on $\mathbb{S}_{N}$ :

$$
\mu_{\phi}(E)=\sigma_{N}\left(\phi^{*^{-1}}(E) \cap \mathbb{S}_{N}\right)
$$

for every Borel subset $E \subset \overline{\mathbb{B}_{N}}$.

We will need a classical criterion of compactness of composition operators on HardyOrlicz spaces. Its proof is an easy adaptation of that of [4, Proposition 3.11].

Proposition 3.1. Let $\psi$ be an Orlicz function and let $\phi: \mathbb{B}_{N} \rightarrow \mathbb{B}_{N}$ be holomorphic. $C_{\phi}$ is compact on $H^{\psi}\left(\mathbb{B}_{N}\right)$ if and only if, for every sequence $\left(f_{n}\right)_{n}$ in the unit ball of $H^{\psi}\left(\mathbb{B}_{N}\right)$ converging to 0 uniformly on every compact subset of $\mathbb{B}_{N}, f_{n} \circ \phi$ converges to 0 in $H^{\psi}\left(\mathbb{B}_{N}\right)$.

Due to the non-separability of small Hardy-Orlicz spaces, the following general theorem will not be a trivial consequence of embedding theorems, as it might be the case for classical Hardy spaces.

Theorem 3.2. Let $\psi$ be an Orlicz function which satisfies the $\nabla_{2}$-Condition and let $\phi$ : $\mathbb{B}_{N} \rightarrow \mathbb{B}_{N}$ be holomorphic.

(1) If $\psi$ satisfies the uniform $\nabla_{0}$-Condition, then $C_{\phi}$ is bounded from $H^{\psi}\left(\mathbb{B}_{N}\right)$ into itself if and only if $\mu_{\phi}$ is a $\psi$-Carleson measure.

(2) If $\psi$ satisfies the $\nabla_{0}$-Condition, then $C_{\phi}$ is compact from $H^{\psi}\left(\mathbb{B}_{N}\right)$ into itself if and only if $\mu_{\phi}$ is a vanishing $\psi$-Carleson measure.

Proof. The difficult part of the theorem is the sufficient part: if $\mu_{\phi}$ is a $\psi$-Carleson measure (resp. vanishing $\psi$-Carleson measure), then, under uniform $\nabla_{0^{-}}$Condition (resp. $\nabla_{0^{-}}$ Condition), $C_{\phi}$ is bounded (resp. compact) on $H^{\psi}\left(\mathbb{B}_{N}\right)$. The converse is quite similar to the proof of 1 ) of Theorem 2.7 (resp. Theorem 2.12), using test functions.

We turn to the proof of the sufficiency in 1). Since $\psi$ satisfies $\nabla_{2}$-Condition, Theorem 1.9 ensures that $\left(H M^{\psi}\left(\mathbb{B}_{N}\right)\right)^{* *}=H^{\psi}\left(\mathbb{B}_{N}\right)$, and therefore that the bi-adjoint of $C_{\phi \mid H M \psi}$ is equal to $C_{\phi}$ itself. Hence, if $C_{\phi}$ is bounded from $H M^{\psi}\left(\mathbb{B}_{N}\right)$ into itself, then it is bounded from $H^{\psi}\left(\mathbb{B}_{N}\right)$ into itself (note that the converse is trivially true, since $C_{\phi}(f) \in H M^{\psi}\left(\mathbb{B}_{N}\right)$ if $f \in H M^{\psi}\left(\mathbb{B}_{N}\right)$, whenever $C_{\phi}$ is bounded on $H^{\psi}\left(\mathbb{B}_{N}\right)$ ). So, for $\psi$ satisfying the uniform 
$\nabla_{0^{-}}$-Condition, it is sufficient to show that, if $\mu_{\phi}$ is a $\psi$-Carleson measure, then $C_{\phi}$ is bounded from $H M^{\psi}\left(\mathbb{B}_{N}\right)$ into itself. Thanks to Theorem 2.7, it is still sufficient to prove that, for any $f \in H M^{\psi}\left(\mathbb{B}_{N}\right),\left\|j_{\mu_{\phi}}(f)\right\|_{\psi}=\left\|C_{\phi}(f)\right\|_{\psi}$, where $j_{\mu_{\phi}}$ is the embedding $H^{\psi}\left(\mathbb{B}_{N}\right) \hookrightarrow L^{\psi}\left(\mu_{\phi}\right)$. Now, it is not difficult to show that

$$
\left\|j_{\mu_{\phi}}(f)\right\|_{\psi}=\left\|C_{\phi}(f)\right\|_{\psi}
$$

for any $f$ in the ball algebra $A\left(\mathbb{B}_{N}\right)$, and (3.1) can be extended to $H M^{\psi}\left(\mathbb{B}_{N}\right)$, by density of $A\left(\mathbb{B}_{N}\right)$ into $H M^{\psi}\left(\mathbb{B}_{N}\right)$, which concludes 1$)$.

The proof of sufficiency for compactness, in 2), follows exactly the same argument as above and uses the fact that, if $\mu_{\phi}$ is a vanishing $\psi$-Carleson measure then, under $\nabla_{0^{-}}$ Condition, $j_{\mu_{\phi}}$ is compact (Theorem 2.12) so that $C_{\phi}$ is compact from $H M^{\psi}\left(\mathbb{B}_{N}\right)$ into itself, because of Proposition 2.11 and Proposition 3.1.

Remark 3.3. If we do not assume that $\psi$ satisfies the uniform $\nabla_{0^{-}}$Condition (resp. $\nabla_{0^{-}}$ Condition), then Theorem 2.7 (resp. Theorem 2.12) provides a priori non-equivalent necessary and sufficient conditions to the boundedness (resp. compactness) of $C_{\phi}$ on $H^{\psi}\left(\mathbb{B}_{N}\right)$.

The following corollary is a particular case of Theorem 3.2:

Corollary 3.4. Let $\psi$ be an Orlicz function which satisfies the $\Delta_{2}$ and $\nabla_{2}$-Condition (i.e. $H^{\psi}\left(\mathbb{B}_{N}\right)$ is reflexive) and let $\phi: \mathbb{B}_{N} \rightarrow \mathbb{B}_{N}$ be holomorphic.

(1) $C_{\phi}$ is bounded from $H^{\psi}\left(\mathbb{B}_{N}\right)$ into itself if and only if $\mu_{\phi}$ is a Carleson measure.

(2) $C_{\phi}$ is compact from $H^{\psi}\left(\mathbb{B}_{N}\right)$ into itself if and only if $\mu_{\phi}$ is a vanishing Carleson measure.

Proof. It suffices to observe that

$$
\frac{1}{\psi\left(A \psi^{-1}(1 / h)\right)} \approx h^{N}
$$

for every $A>0$, whenever $\psi$ is an Orlicz function which satisfies the $\Delta_{2}$-Condition (see Remark 2 (a) following Theorem 4.11 in [9]). In particular, this allows not to assume that $\psi$ satisfies the $\nabla_{0}$-Condition.

A first consequence of the previous results is the following corollary:

Corollary 3.5. Let $\phi: \mathbb{B}_{N} \rightarrow \mathbb{B}_{N}$ be holomorphic and let $\psi, \nu$ be two Orlicz functions which both satisfy $\nabla_{2}$-Condition. We assume that $\nu$ also satisfies the $\Delta_{2}$-Condition. Then

(1) If $C_{\phi}$ is bounded on $H^{\nu}\left(\mathbb{B}_{N}\right)$ (e.g. on any $H^{p}\left(\mathbb{B}_{N}\right)$ ), then it is bounded on $H^{\psi}\left(\mathbb{B}_{N}\right)$;

(2) If in addition $\psi$ satisfies the $\nabla_{0}$-Condition and if $C_{\phi}$ is compact on $H^{\psi}\left(\mathbb{B}_{N}\right)$, then it is compact on $H^{\nu}\left(\mathbb{B}_{N}\right)$ (e.g. on any $H^{p}\left(\mathbb{B}_{N}\right)$ ).

Proof. The first point follows from Remark 3.3 and from the fact that if $\mu$ is a Carleson measure, i.e. if $K_{\mu} \leq C$ for some constant $C \geq 1$, then $\mu$ satisfies Condition (2.11) for some $0<A \leq 1$.

For the second point, it suffices to show that Condition (2.17) implies that $\mu$ is a vanishing Carleson measure, what is trivial if we apply it for $A=1$.

One of the main motivations of this paper is to investigate whether there exist some Hardy-Orlicz spaces on which composition operators are always bounded. To this purpose, we will use a result, which is mentionned in [11], yet not proven: 
Proposition 3.6. If $\phi: \mathbb{B}_{N} \rightarrow \mathbb{B}_{N}$ is holomorphic, then there exists a constant $B>0$ such that

$$
\mu_{\phi}(\mathcal{S}(\xi, h)) \leq B . h
$$

uniformly with respect ot $\xi \in \mathbb{S}_{N}$ and for every $0<h<1$.

To be more precise, in their paper, B. MacCluer and P. Mercer proved a quite similar result, where $\mu_{\phi}$ is the pull-back measure by $\phi$ of the weigthed Lebesgue volume measure $v_{\alpha}$ on strictly pseudo-convex domains. Since we did not find the proof of the above proposition written anywhere, we prefer to give it here:

Proof. We fix $\xi \in \mathbb{S}_{N}$ and $0<h<1$. We denote by $\chi_{\left(\phi^{*}\right)^{-1}(\mathcal{S}(\xi, h))}$ the characteristic function of $\left(\phi^{*}\right)^{-1}(\mathcal{S}(\xi, h))$. The formula of integration by slices ([15, Proposition 1.4.7, (1)]) yields

$$
\begin{aligned}
\mu_{\phi}(\mathcal{S}(\xi, h)) & =\int_{\mathbb{S}_{N}} \chi_{\left(\phi^{*}\right)^{-1}(\mathcal{S}(\xi, h))}(\zeta) d \sigma_{N}(\zeta) \\
& =\int_{\mathbb{S}_{N}} \int_{\mathbb{T}} \chi_{\left(\phi^{*}\right)^{-1}(\mathcal{S}(\xi, h))}(u \zeta) d \lambda(u) d \sigma_{N}(\zeta),
\end{aligned}
$$

where $\lambda$ is the Lebesgue measure on the torus $\mathbb{T}$. Let us observe that $\chi_{\left(\phi^{*}\right)^{-1}(\mathcal{S}(\xi, h))}(u \zeta)=1$ is equivalent to

$$
\left|1-\left\langle\phi^{*}(u \zeta), \xi\right\rangle\right|<h .
$$

For every $\zeta \in \mathbb{S}_{N}$, let $\varphi_{\zeta, \xi}: \mathbb{D} \rightarrow \mathbb{D}$ be the function defined by $\varphi_{\zeta, \xi}(z)=\langle\phi(z \zeta), \xi\rangle$ for any $z \in \mathbb{D} . \varphi_{\zeta, \xi}$ is holomorphic and it is not difficult to verify that $\varphi_{\zeta, \xi}^{*}(u)=\left\langle\phi^{*}(u \zeta), \xi\right\rangle$ for $\lambda$-almost every $u \in \mathbb{T}$, where $\varphi_{\zeta, \xi}^{*}$ is the $\lambda$-almost everywhere radial limit of $\varphi_{\zeta, \xi}$. Inequality (3.3) is then equivalent to $\varphi_{\zeta, \xi}^{*}(u) \in \mathcal{S}_{1}(1, h)$, where $\mathcal{S}_{1}(1, h)$ is the one-dimensional disk of radius $h$, centered at 1 , intersected with $\mathbb{D}$. Now, by the Littlewood subordination principle together with the classical (automatic) characterization of boundedness of $C_{\phi}$ on $H^{p}(\mathbb{D})$, there exists a constant $B>0$, independent of $\zeta$ and $\xi$, such that

$$
\lambda\left(\left(\varphi_{\zeta, \xi}^{*}\right)^{-1}\left(\mathcal{S}_{1}(1, h)\right)\right) \leq B . h
$$

which concludes the proof.

Now, if we compare Condition (3.2) and Condition (2.10), written for $\psi_{1}=\psi_{2}=\psi$, it clear that, if we can find some $\psi$, among those satisfying the uniform $\nabla_{0^{-}}$Condition, which satisfies the following condition $\mathcal{P}$ :

$\mathcal{P}$ : for every $K>0$, there exist $A>0$ and $h_{0}>0$ such that

$$
K h \leq \frac{1}{\psi\left(A \psi^{-1}\left(1 / h^{N}\right)\right)},
$$

for any $0<h \leq h_{0}$,

then every composition operator will be bounded on the Hardy-Orlicz space $H^{\psi}\left(\mathbb{B}_{N}\right)$, according to Theorem 3.2. The next proposition characterizes those Orlicz functions which satisfy this condition $\mathcal{P}$ :

Proposition 3.7. Let $\psi$ be an Orlicz function. $\psi$ satisfies Condition $\mathcal{P}$ if and only if, for every $K>0$ (or equivalently for one $K>0$ ), there exists $C>0$ such that, for every $x>0$ large enough, we have

$$
\psi(x)^{N} \leq K \psi(C x) .
$$

In particular, Condition $\mathcal{P}$ is trivial if $N=1$ and coincides with $\Delta^{2}$-Condition whenever $N>1$. 
Proof. The first part comes from a straightforward rewritening of Inequality (3.4). The second part is a direct application of Proposition 1.3, using convexity of $\psi$.

When $N=1$, [8, Theorem 4.19] permits to remove the necessary uniform $\nabla_{0}$-Condition in the the first point of Theorem [3.2. When $N>1$, this trick fails as it is not difficult to check that if this result could be extended to the several complex variables setting, then it would imply that every composition operator is bounded on $H^{p}\left(\mathbb{B}_{N}\right)$. Moreover, thanks to the factorization of a function $f \in H^{\psi}(\mathbb{D})$ (hence in $H^{1}(\mathbb{D})$ ) by a Blaschke product formed with its zeros and a non-zeros function in $H^{\psi}(\mathbb{D})$ (see the remark which precedes the proof of [9, Theorem 4.10], and [2, Section 7, Theorem 1.1]), it is also possible to remove the $\nabla_{2}$-Condition, when $N=1$. Nevertheless, this argument cannot still be extended to $N>1$. Yet, we know (Proposition 1.5) that every Orlicz function satisfying the $\Delta^{2}$-Condition satisfies the uniform $\nabla_{0}$-Condition, and then the $\nabla_{2}$-Condition too.

Therefore, Theorem 3.2, Proposition 3.6 and Proposition 3.7 immediately yields the following result:

Theorem 3.8. Let $\psi$ be an Orlicz function.

(1) Every composition operator is bounded from $H^{\psi}(\mathbb{D})$ into itself;

(2) When $N>1$, if $\psi$ satisfies the $\Delta^{2}$-Condition, then every composition operator is bounded from $H^{\psi}\left(\mathbb{B}_{N}\right)$ into itself.

Acknowledgements. I am grateful to Frédéric Bayart, Alexander Borichev and Daniel Li for their relevent comments and suggestions.

\section{REFERENCES}

[1] A. B. Alexandrov, Existence of inner functions in the unit ball, Math. USSR Sbornik 46 (1983), 143-159.

[2] M. Andersson, Topics in Complex Analysis, Springer Verlag, New York, 1997.

[3] S. Charpentier, Composition operators on Bergman-Orlicz spaces, preprint.

[4] C. C. Cowen, B. D. MacCluer, Composition Operators on Spaces of Analytic Functions, Studies in Advanced Mathematics, CRC Press (1995).

[5] N. Dunford, J. T. Schwartz, Linear operators, I: General Theory, Pure and Appl. math., vol. 7 , Interscience, New York (1958).

[6] M. A. Krasnoselśkit, Ya. B. Rutickit, Convex functions and Orlicz spaces, P. Noordhoff Ltd., Groningen (1961).

[7] P. Lefèvre, D. Li, H. Queffélec, L. Rodríguez-Piazza, Compact composition operators on $H^{2}(\mathbb{D})$ and Hardy-Orlicz spaces, J. Math. Anal. Appl. 354 (2009), 360-371.

[8] P. Lefèvre, D. Li, H. Queffélec, L. Rodríguez-PiazzA, Composition operators on BergmanOrlicz spaces, hal-00426831 (2009).

[9] P. Lefìvre, D. Li, H. Queffélec, L. Rodríguez-Piazza, Composition operators on Hardy-Orlicz spaces, Mem. Am. Math. Soc. 207 (2010), No. 974.

[10] P. Lefèvre, D. Li, H. Queffélec, L. Rodríguez-PiazzA, Some revisited results about composition operators on Hardy spaces, hal-00448623, (2010).

[11] B. D. MacCluer, P. R. Mercer, Composition Operators Between Hardy and Weighted Bergman Spaces on Convex Domains in $\mathbb{C}^{n}$, Proc. Am. Math. Soc. 123 (1995), No. 7, 2093-2102.

[12] B. D. MacCluer, J. H. Shapiro, Angular derivatives and compact composition operators on the Hardy and Bergman spaces, Canad. J. Math. 38 (1986), No. 4, 878-906.

[13] S. C. Power, Hörmander's Carleson theorem for the ball, Glasgow Math. J. 26 (1985), 13-17.

[14] M. M. Rao, Z. D. Ren, Theory of Orlicz spaces, Pure and Applied Mathematics 146, Marcel Dekker, Inc. (1991).

[15] W. Rudin, Function Theory in the Unit Ball of $\mathbb{C}^{n}$, Springer-Verlag, New York, 1980.

[16] J. H. Shapiro, Composition operators and classical function theory, Universitext. Tracts in Mathematics, Springer-Verlag, New York (1993). 
[17] K. ZHu, Spaces of Holomorphic Functions in the Unit Ball, Graduate Texts in Mathematics, Springer (2005).

Charpentier Stéphane, Département de Mathématiques, Bâtiment 425, Université ParisSud, F-91405, Orsay, France

E-mail address: stephane.charpentier@math.u-psud.fr 